\title{
Fuzzy Word Similarity: A Semantic Approach Using WordNet
}

\author{
Sukanya Manna and B. Sumudu U. Mendis
}

\begin{abstract}
In this paper we present a hybrid measure of semantic word similarity using fuzzy inference system which combines both the corpus based distance measures as well as gloss overlap to get the final similarity between two words. We use WordNet as a lexical dictionary to get semantic information about words. We show that this new measure reasonably correlates to human judgments and the average performance is boosted by using triangular membership function in the output.
\end{abstract}

\section{INTRODUCTION}

This paper introduces fuzzy word similarity, which is a measure of semantic relatedness that is based on information from a machine readable dictionary. In particular, this measure takes advantage of hierarchies or taxonomies of concepts as found in resources such as the lexical database as WordNet [1]. Concepts rather words are commonly represented in dictionaries by word senses, each of which has a definition or gloss that briefly describes its meaning. Our measure determines how much related two words are by combining two different kinds of measures of relatedness through a fuzzy information system; using information content of edge based method and gloss overlap of the words being considered. These related words are explicitly encoded in WordNet as relations like 'is-a-kind-of', or 'is-a-part-of', but can be found in any dictionary via synonyms, antonyms and so on.

Fuzzy logic deals with approximate reasoning having vague or imprecise information such as human decision making process which has certain degree of uncertainty in them. Since words are context sensitive, there is often uncertainty associated in finding their similarity. It depends on how a person understands a topic based on the senses of word. So when automating this process, there is also greater chance that different methods will provide different similarity values for same pair of word. To overcome this difficulty of different algorithms, we use fuzzy inference system to analyze two different kinds of measures of word similarity to find a proper way of defining the similarity closer to human judgements.

This paper begins with some related works on some popular word similarity measures. Then we provide a brief description of WordNet, which is used in developing our measure, followed by the basic outline of fuzzy inference system. Then we present our fuzzy word similarity measure, experiments and evaluations.

\footnotetext{
The authors are with the Information and Human Centered Computing group, School of Computer Science, The Australian National University, ACT 0200, Australia; email:\{sukanya.manna, sumudu.mendis\}@anu.edu.au
}

\section{Measuring Semantic Similarity of wORDS}

Measuring word similarity, in terms of utilizing knowledge base, can be broadly classified into knowledge-rich and knowledge-poor methods [2], [3]. The knowledge-rich approaches require either a conceptual dependency representation, or semantic tagging of the words. Most methods that calculate semantic distance through WordNet [1] or Rogets thesaurus fall into this category; while the knowledgepoor approaches require no previously encoded semantic information, and depend on frequency of co-occurrence of word contexts to determine similarity. It may be further categorized according to how co-occurrence frequency data is handled [4]:

1) Vector space: In this case, the basic assumption lies in the fact that semantically related words are more likely to co-occur in the corpus. A matrix is then constructed in word-by-word or word-by-document order where each cell contains value such as term frequency (TF) or $\mathrm{TF}^{*} \mathrm{IDF}$ (inverse document frequency) or any other kind of weighted frequency measure. Word similarity is hence established by comparing distance measures such as the cosine coefficient or Euclidean distance.

2) Syntactic parsing: Suppose that the semantic relatedness of words leads to their use in similar grammatical structures in their contexts. Judging word similarity is achieved by tagging parts-of-speech in the corpus, shallow parsing of sentences, specifying the relationship between chunks and comparing the syntactic components along with their dependency relations [2].

Now let us here describe some of the semantic relatedness measures which have been proposed in recent years. Most of them rely on the noun taxonomy of the lexical database WordNet. It is seen that the popular methodologies for measuring semantic relatedness with the help of a thesaurus can be classified into three categories: one uses the solely semantic links (i.e. edge-counting), the other combines corpus statistics with the taxonomic distance and the third one is based on the commonality of information in glosses of the words in the WordNet entry.

1) Edge-counting methods: The edge-counting or shortest path method derives from the geometric model in Cognitive Psychology, where the shorter distance entails the stronger association between stimuli and response. It can be traced back to Quillians semantic memory model [5], [6] where concept nodes are planted within the hierarchical network and the number of hops between the nodes specifies the similarity of the concepts. Then Wu and Palmer [7], [8] proposed to measure the verbal concept similarity in the projected domain hierarchy when translating from English 
verbs to Chinese. According to the claims of $\mathrm{Wu}$ and Palmer, the relatedness of two words is the weighted sum of all their senses comparison. After this, Leacock and Chodorow [9] adopted the concept of information content [10] in part to evaluate the relatedness of two words using the following model: $\operatorname{Sim}\left(W_{i}, W_{j}\right)=\left[-\log \frac{\operatorname{Dist}\left(c_{i}, c_{j}\right.}{2 \times D}\right]$, where $\operatorname{Dist}\left(c_{i}, c_{j}\right)$ is the shortest distance between concepts $c_{i}$ and $c_{j} ; D$ is the maximum depth of the taxonomy.

2) Corpus based methods: Resnik [10] augments each synset in WordNet with an information content value derived from a large corpus of text. The measure of relatedness between two concepts is taken to be the information content value of the most specific concept that the two concepts have in common. He defines information content of nearest common node (nch) to explain the similarity of two words through frequency statistics retrieved from a corpus, not through the distance of edge-counting. Here the frequency of ncn subsumes all the frequency data of subordinate concept nodes. The information content can be quantified as the negative of the $\log$ likelihood, $-\log \mathrm{P}(\mathrm{c})$. However, Resnik still employs the structure of a conceptual net and one drawback is that the $n c n$ for all concept pairs that have the same parent node is the same. [11] and [12] extend Resniks measure by scaling the common information content values by those of the individual concepts.

3) Gloss based measures: Lesk [13] defines relatedness in terms of dictionary definition overlaps of concepts. He describes an algorithm that disambiguates words based on the extent of overlaps of their dictionary definitions with those of words in the context. The sense of the target word with the maximum overlaps is selected as the assigned sense of the word. The hypothesis that dictionary definition overlaps can measure semantic relatedness underlies this algorithm. Banerjee and Pedersen [14] adapt the Word Sense Disambiguation algorithm, described by Lesk [13], to WordNet. Since the Lesk algorithm was designed before the creation of WordNet, it was mainly based on traditional dictionaries. Banerjee and Pedersen enhance the Lesk algorithm with the rich source of knowledge present in WordNet. The algorithm proceeds by taking each of the words in the context of the ambiguous word and considers the glosses of all words connected to these by various WordNet relations. The overlap of each of these glosses with glosses of words connected to each sense of the ambiguous word is determined and is used to compute a score for these senses. The sense with the highest score is the selected.

\section{BRIEF DESCRIPTION OF WORDNET}

WordNet is a lexical online database for the English language [1]. It groups English words into sets of synonyms called synsets, provides short, general definitions (called glosses), and records the various semantic relations between these synonym sets. The main purpose is to produce a combination of dictionary and thesaurus that is more intuitively usable, and to support automatic text analysis and artificial intelligence applications.
WordNet distinguishes between nouns, verbs, adjectives and adverbs. Every synset contains a group of synonymous words or collocations; different senses of a word are represented in the form of different synsets. Each synset is provided with a short meaning called gloss. Most synsets are connected to other synsets via a number of semantic relations. These relations vary based on the type of word being considered, and can be hypernym, hyponym, holonym, meronym, and troponym.

WordNet also provides the polysemy count of a word i.e., the number of synsets that contain the word. If a word participates in several synsets (i.e. has several senses) then typically some senses are much more common than others. WordNet quantifies this by the frequency score: in which several sample texts have all words semantically tagged with the corresponding synset, and then a count provided indicating how often a word appears in a specific sense.

For any kind of semantic analysis job, researchers either use WordNet or Rojet's thesaurus mainly to formulate their models.

\section{BRIEF OVERVIEW OF FUZZY INFERENCE SYSTEM}

Fuzzy inference is the process of formulating the mapping from a given input to an output using fuzzy logic. The mapping then provides a basis from which decisions can be made, or patterns discerned. The process of fuzzy inference involves: membership functions, fuzzy logic operators, and if-then rules. Fuzzy inference systems (FIS) have been successfully applied in different areas such as automatic control, data classification, decision analysis, expert systems, and computer vision and so on. FIS generally requires three basic steps to compute the fuzzy inference process.

a) Fuzzification: The fuzzification comprises the process of transforming crisp values into grades of membership for linguistic terms of fuzzy sets. The membership function is used to associate a grade to each linguistic term.

b) Rule-base for decision making: Fuzzy rules are linguistic IF-THEN- constructions that have the general form "IF A THEN B" where A and B are (combination of) propositions containing linguistic variables. A is called the antecedent and B is the consequence of the rule. In effect, the use of linguistic variables and fuzzy IF-THEN-rules exploits the tolerance for imprecision and uncertainty. In this respect, fuzzy logic mimics the crucial ability of the human mind to summarize data and focus on decision-relevant information. Once the inputs are fuzzified, the degree to which each part of the antecedent has been satisfied for each rule is known. If the antecedent of a given rule has more than one part, the fuzzy operator is applied to obtain one number that represents the result of the antecedent for that rule. This number will then be applied to the output function. A consequent of a rule is a fuzzy set represented by a membership function. In this step, the consequent is reshaped using a function associated with the antecedent. Since decisions are based on all the rules in a fuzzy inference system, the rules must be combined in some manner in order to make a decision. Aggregation is the 


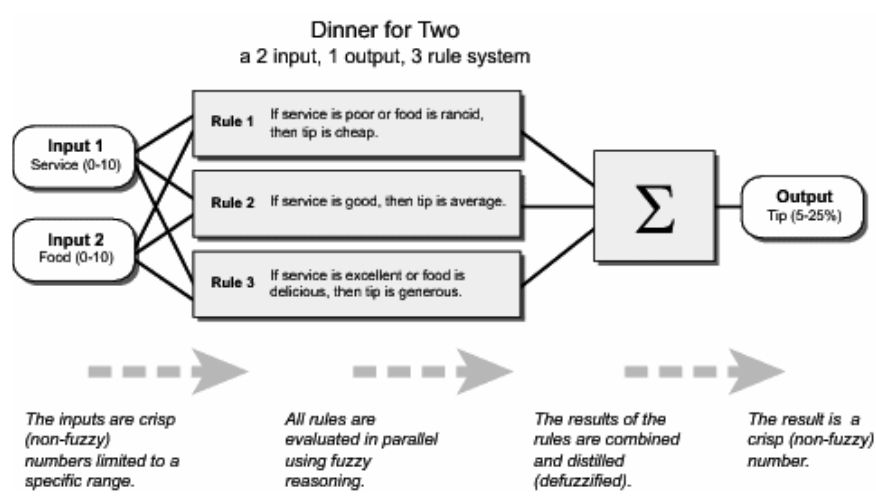

Fig. 1. Example of FIS

process by which the fuzzy sets that represent the outputs of each rule are combined into a single fuzzy set.

c) Defuzzification: Taking fuzzy sets as input, defuzzification process outputs a crisp value, which is suitable for analysis and control. There mainly two significant types of fuzzy inference systems differing in defuzzification part, which are Mamdani-type [15] and Sugeno-type [16]. Mamdani-type inference expects the output membership functions to be fuzzy sets. After the aggregation process, there is a fuzzy set for each output variable that needs defuzzification. Rather than integrating across functions to find defuzzified output, it is also possible to use the weighted average of a few data points. Sugeno-type systems support this type of model. In general, Sugeno-type systems can be used to model any inference system in which the output membership functions are either linear or constant.

4) Example: Here we present an example of how FIS works with a block diagram as shown in fig. $1^{1}$; showing the variation of tips based on the quality of service. The output is the amount of tips to be given. In the middle block, the conditions are framed; based on that, the output is generated after aggregating.

\section{METHOd OF FUZZY WORD SIMILARITY}

In this section, we present our fuzzy word similarity measure. We use two different similarity measures (gloss overlap and path based measure) as input to our fuzzy inference system and as an output we have PGMeasure, where information of both the types are fused based on the rule base created for the similarity scores.

\section{A. Using glosses of related senses}

Word forms from the definitions ("glosses") in WordNet's synsets are manually linked to the context-appropriate sense in WordNet. In sec.II, we have seen that in [13] and [14], they have used glosses to find similarity between concepts. Here, we used Lesk's [13] ideas but computed Jaccard's coefficient of only glosses among different sentences of word pair considered to find semantic similarity between them.

\footnotetext{
${ }^{1}$ The diagram is taken from: http://www.mathworks.com/ access/helpdesk/help/toolbox/fuzzy/fp351dup8.html
}

Let us consider two words $w_{i}^{p}, w_{j}^{p} \in W$, and $p \in P$; where $W$ is the set of words, and $P$ is a set of parts of speech like noun, verb, adjective, and adverb respectively. Let word $w_{i}^{p}$ has $|K|$ synsets and $w_{j}^{p}$ has $|L|$ synsets which can be represented by $S_{k}^{w_{i}^{p}}$ and $S_{l}^{w_{j}^{p}}$ respectively, $k \in[1,|K|]$ and $l \in[1,|L|]$. Now we calculate gloss overlap as $\operatorname{sim}_{\text {gloss }}$ by

$$
\operatorname{sim}_{\text {gloss }}\left(w_{i_{k}}^{p}, w_{j_{l}}^{p}\right)=\operatorname{JaccardCoef}\left(S_{k}^{w_{i}^{p}}, S_{l}^{w_{j}^{p}}\right)
$$

\section{B. Path based similarity scores}

In this section we present two different types of word similarities which we have used for evaluation of our method. Both of these methods are corpus based approaches but also uses edge/path information for finding the similarity between two concepts present in the taxonomical hierarchy of any kind of lexical dictionary.

1) Jiang and Conraths model: Jiang and Conrath [11] proposed a measure which incorporates the information content of the two concepts, along with that of their lowest common subsumer (lcs). The measure is a distance measure that specifies the extent of unrelatedness of two concepts. It combines features of simple edge counting with those of information content introduced in the Resnik [10]. They also considered the link type, depth, conceptual density, and information content of concepts. Their simplified formula can be expressed as follows:

$$
\begin{gathered}
\operatorname{Dist}\left(c_{i}, c_{j}\right)=\operatorname{IC}\left(c_{i}\right)+\operatorname{IC}\left(c_{j}\right) 2 * \operatorname{IC}\left(\operatorname{lcs}\left(c_{i}, c_{j}\right)\right) \\
\operatorname{Sim}\left(c_{i}, c_{j}\right)=\operatorname{Dist}\left(c_{i}, c_{j}\right)
\end{gathered}
$$

where $c_{i}$ and $c_{j}$ are two concepts whose relatedness we are tending to find, $I C$ determines the information content of a concept and $l c s\left(c_{i}, c_{j}\right)$ finds the lowest common subsuming concept of concepts $c_{i}$ and $c_{j}$.

Now in our case, we replace the concepts $c_{i}, c_{j}$ by words $w_{i}^{p}, w_{j}^{p}, i, j \in W$, and $p \in P$; where $W$ is the set of words, and $P$ is a set of parts of speech like noun, verb, adjective, and adverb respectively. Let word $w_{i}^{p}$ has $|K|$ synsets and $w_{j}^{p}$ has $|L|$ synsets which can be represented by $S_{k}^{w_{i}^{p}}$ and $S_{l}^{w_{j}^{p}}$ respectively, $k \in[1,|K|]$ and $l \in[1,|L|]$. We named this measure as path measure as $\operatorname{sim}_{\text {path }}$ by

$$
\operatorname{sim}_{\text {path }}\left(w_{i_{k}}^{p}, w_{j_{l}}^{p}\right)=\text { related }_{J C}\left(S_{k}^{w_{i}^{p}}, S_{l}^{w_{j}^{p}}\right)
$$

2) Lin's model: Lin [12] used a similarity model, which uses edge counting method as well as information content of the concepts to find the similarity. So, we rather prefer calling it to be path measure as it exploits taxonomical hierarchy of the WordNet as well as corpus based statistical measure for computation. This can be represented as,

$$
\text { related }_{l i n}\left(c_{i}, c_{j}\right)=\frac{2 . I C\left(l c s\left(c_{i}, c_{j}\right)\right)}{I C\left(c_{i}\right)+I C\left(c_{j}\right)}
$$

where $c_{i}$ and $c_{j}$ are two concepts whose relatedness we are tending to find, $I C$ determines the information content of a 
TABLE I

CO-ORDINATES OF DIFFERENT MEMBERSHIP FUNCTIONS OF FUZZY SETS USED HERE

\begin{tabular}{c|c|c|c|c}
\hline Low_trapez & $(0,0)$ & $(0,1)$ & $(0.3,1)$ & $(0.4,0)$ \\
Medium_trapez & $(0.3,0)$ & $(0.4,1)$ & $(0.6,1)$ & $(0.7,0)$ \\
High_trapez & $(0.6,0)$ & $(0.8,1)$ & $(1,1)$ & $(0,1)$ \\
\hline \hline Low_tri & $(0,0)$ & $(0.3,1)$ & $(0.6,0)$ & - \\
Medium_tri & $(0.3,0)$ & $(0.55,1)$ & $(0.8,0)$ & - \\
High_tri & $(0.6,0)$ & $(0.8,1)$ & $(1,0)$ & - \\
\hline
\end{tabular}

concept and $l c s\left(c_{i}, c_{j}\right)$ finds the lowest common subsuming concept of concepts $c_{i}$ and $c_{j}$.

Now in our case, we replace the concepts $c_{i}, c_{j}$ by words $w_{i}^{p}, w_{j}^{p} \in W$, and $p \in P$; where $W$ is the set of words, and $P$ is a set of parts of speech like noun, verb, adjective, and adverb respectively. Let word $w_{i}^{p}$ has $|K|$ synsets and $w_{j}^{p}$ has $|L|$ synsets which can be represented by $S_{k}^{w_{i}^{p}}$ and $S_{l}^{w_{j}^{p}}$ respectively, $k \in[1,|K|]$ and $l \in[1,|L|]$. We named this measure as path measure as $\operatorname{sim}_{\text {path }}$ by

$$
\operatorname{sim}_{\text {path }}\left(w_{i_{k}}^{p}, w_{j_{l}}^{p}\right)=\text { related }_{l i n}\left(S_{k}^{w_{i}^{p}}, S_{l}^{w_{j}^{p}}\right)
$$

\section{Combined Path-Gloss measure (PGMeasure) using FIS}

We combine Path measure, especially the measure proposed by Lin et al., and Gloss overlap to create PGMeasure using FIS. For computation of this, we create two input fuzzy sets; one for path measure and the other for gloss overlap with three membership functions in each case based on low similarity, medium similarity, and high similarity, signifying to what extent two words are similar to each other.

1) Determination of input fuzzy sets: We use same notations for words as used in the previous equations (1) and (6). Now, let us consider, two input fuzzy sets, pathMeasure and glossOverlap. We used trapezoidal membership functions of low $(L)$, medium $(M)$, and high $(H)$ in both the cases. The co-ordinates $(x, y)$ for the trapezoidal membership functions are shown in table.I.

For pathMeasure we denote three membership values low, medium, and high as $\mu_{L}\left(\operatorname{sim}_{\text {path }}\left(w_{i_{k}}^{p}, w_{j_{l}}^{p}\right)\right)$, $\mu_{M}\left(\operatorname{sim}_{\text {path }}\left(w_{i_{k}}^{p}, w_{j_{l}}^{p}\right)\right)$, and $\mu_{H}\left(\operatorname{sim}_{\text {path }}\left(w_{i_{k}}^{p}, w_{j_{l}}^{p}\right)\right) \in$ $[0,1] . \operatorname{sim}_{\text {path }}\left(w_{i_{k}}^{p}, w_{j_{l}}^{p}\right)$ is computed using (6) or (4).

Likewise, for the fuzzy set glossOverlap, we define three membership values low, medium, and high as $\mu_{L}\left(\operatorname{sim}_{\text {gloss }}\left(w_{i_{k}}^{p}, w_{j_{l}}^{p}\right)\right), \quad \mu_{M}\left(\operatorname{sim}_{\text {gloss }}\left(w_{i_{k}}^{p}, w_{j_{l}}^{p}\right)\right)$, and $\mu_{H}\left(\operatorname{sim}_{\text {gloss }}\left(w_{i_{k}}^{p}, w_{j_{l}}^{p}\right)\right) \in[0,1] . \operatorname{sim}_{\text {gloss }}\left(w_{i_{k}}^{p}, w_{j_{l}}^{p}\right)$ is computed using (1).

2) Fuzzy rule-bases: In fuzzy system, there are different rule extraction methods as seen in [17] besides expert knowledge. We present in fig. 2 the rules generated for our FIS. The rules are decided manually based on expert's understanding about the similarity measures.

3) Determining output fuzzy set: We used triangular membership functions as output fuzzy set. Triangular is used over trapezoidal in order to overcome some practical issues in the 'centre of gravity (COG)' calculation approximation in

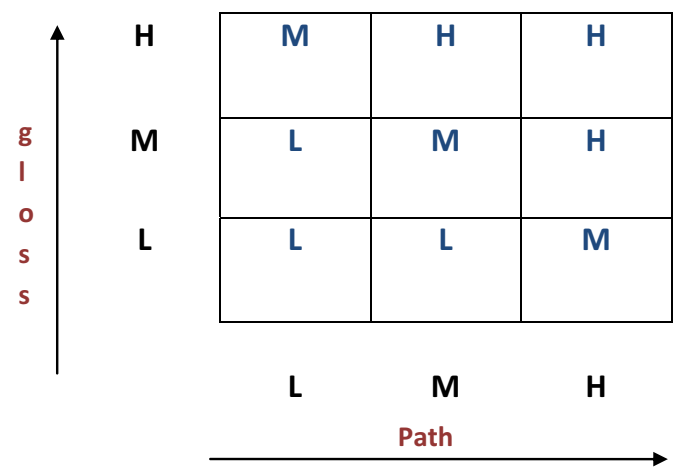

Fig. 2. Rules for FIS for obtaining PGMeasure of word similarity

the latter i.e, if both the inputs are 1 then we expect the output value to be 1 as well. But due to approximation of COG in trapezoidal function, it gives the value less than 1. We can avoid this issue using triangular function in output. Now, $\mu_{L}\left(\operatorname{sim}_{P G}\left(w_{i_{k}}^{p}, w_{j_{l}}^{p}\right)\right), \mu_{M}\left(\operatorname{sim}_{P G}\left(w_{i_{k}}^{p}, w_{j_{l}}^{p}\right)\right)$, and $\mu_{H}\left(\operatorname{sim}_{P G}\left(w_{i_{k}}^{p}, w_{j_{l}}^{p}\right)\right), \mu\left(\operatorname{sim}_{P G}\left(w_{i_{k}}^{p}, w_{j_{l}}^{p}\right)\right) \in[0,1]$ are the corresponding membership functions for the output fuzzy set PGMeasure. The co-ordinates of the triangular membership functions are defined in table.I. Now, $\left.\operatorname{sim}_{P G}\left(w_{i_{k}}^{p}, w_{j_{l}}^{p}\right)\right)$ is the defuzzified output of the the PGMeasure FIS for all combination of synsets of the word pairs $w_{i}^{p}$ and $w_{j}^{p}$.

Now min - max inference is computed across the cartesian products of the input fuzzy sets using the rule-base shown in fig.2. Next, $\left.\operatorname{sim}_{P G}\left(w_{i_{k}}^{p}, w_{j_{l}}^{p}\right)\right)$ is the defuzzified output obtained using 'Centre of Gravity' method.

Thus, the final fuzzy word similarity score of a word pair is hence computed by,

$$
\left.\operatorname{sim}_{P G M \text { easure }}\left(w_{i}^{p}, w_{j}^{p}\right)=\operatorname{Max}\left[\operatorname{sim}_{P G}\left(w_{i_{k}}^{p}, w_{j_{l}}^{p}\right)\right)\right]
$$

which is the maximum of all the defuzzified values of all possible combinations of synsets of the pair of words considered.

\section{Example}

We present here an example which explains stepwise computation of our whole process of obtaining PGMeasure. In Fig.3, is an example of the input fuzzy sets representing path and gloss similarity measures, having trapezoidal membership functions in each case. In this example, we have deliberately taken trapezoidal membership function to show centre of gravity problem in extremities. The figures are self explanatory, so we do not describe the thresholds of the membership functions explicitly. Now, to illustrate the min-max inference, we will go through step-by-step with a hypothetical example. Suppose the path and gloss scores are as follows:

path $=0.25 \Rightarrow($ Low, 0.6), (Medium, 0.4), (High, 0.0) gloss $=0.75 \Rightarrow$ (Low, 0.0), (Medium, 0.25), (High, 0.75)

By taking all possible combinations of Low, Medium and High for path and gloss, we determine the outcome of each by following the rule base in fig. 2 and taking the minimum 


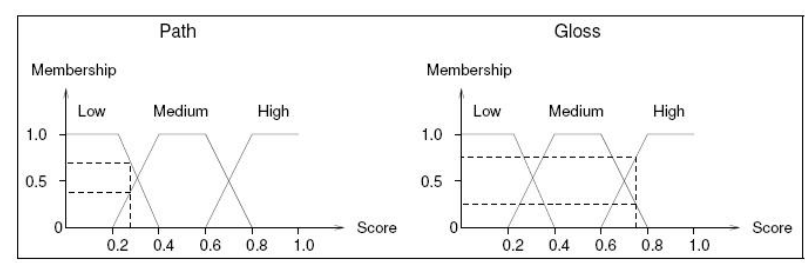

Fig. 3. Example path and gloss overlap

$$
\begin{array}{rrr}
(L, L) \rightarrow \quad(\operatorname{rule}(L, L), \min (0.6,0.0))= & (L, 0.0) \\
(L, M) \rightarrow \quad(\operatorname{rule}(L, M), \min (0.6,0.25))= & (L, 0.25) \\
(L, H) \rightarrow \quad(\operatorname{rule}(L, H), \min (0.6,0.75))= & (M, 0.6) \\
(M, L) \rightarrow \quad(\operatorname{rule}(M, L), \min (0.4,0.0))= & (L, 0.0) \\
(M, M) \rightarrow \quad(\operatorname{rule}(M, M), \min (0.4,0.25))= & (M, 0.25) \\
(M, H) \rightarrow \quad(\operatorname{rule}(M, H), \min (0.4,0.75))= & (H, 0.4) \\
(H, L) \rightarrow \quad(\operatorname{rule}(H, L), \min (0.0,0.0))= & (M, 0.0) \\
(H, M) \rightarrow \quad(\operatorname{rule}(H, M), \min (0.0,0.25))= & (H, 0.0) \\
(H, H) \rightarrow \quad(\operatorname{rule}(H, H), \min (0.0,0.75))= & (H, 0.0)
\end{array}
$$

Fig. 4. Example of rule triggering

membership value within in each pair. $(\mathrm{M}, \mathrm{H})$ for instance represents the outcome of the Path score with membership Medium and Gloss score with membership High. From above, the path score of 0.25 has membership of 0.4 to Medium and the gloss score of 0.75 has a High membership of 0.75. Following the rule base in fig.2, we can see Medium from Path and High from Gloss $\Rightarrow$ High output PGMeasure. So we say $(\mathrm{M}, \mathrm{H}) \Rightarrow(\mathrm{H} ; 0.4)$ where 0.4 is the minimum of 0.4 and 0.75 . Thus a fully worked solution for the example would look like fig.4. Now, the maximum values for each set are taken. So for the set Low, we take $\max (0.0 ; 0.25 ; 0.0)$, and so on. The final result should look something like: (L, 0.25), (M, 0.25), (H, 0.4).

The Center of Gravity method is easy to understand by referring to fig.5. In this example, suppose we want to get the crisp value of relatedness between particular senses two verbs. Given the fuzzy values of $(\mathrm{L}, 0.3),(\mathrm{M}, 0.7),(\mathrm{H}, 0.4)$, we shade the sets corresponding to their membership value (as shown in fig.5). The center of gravity is given by finding the center of the shaded area, and reading the corresponding value for score. In this case it corresponds to a value around 0.6. This method however is not perfect. The method has problems at the extremities. The center of gravity for instance of (L, 0.0), (M, 0.0), (H, 1.0) which should correspond to the best possible score for word similarity, in fact gives a score of around 0.85 as seen in fig.6. A similar problem also exists for the fuzzy input set $(\mathrm{L}, 1.0),(\mathrm{M}, 0.0),(\mathrm{H}, 0.0)$. Therefore we used triangular membership function to overcome this problem.

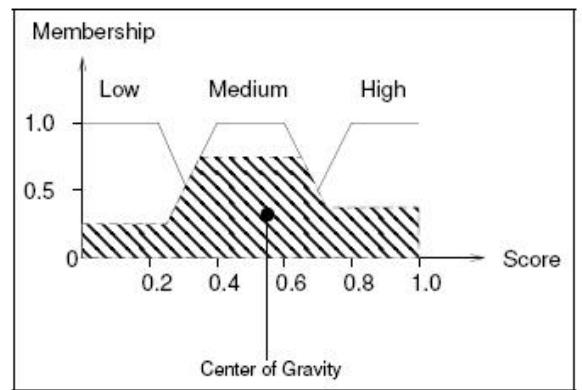

Fig. 5. Example showing COG

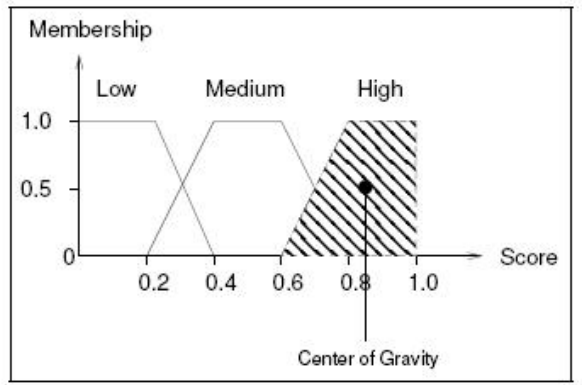

Fig. 6. Example showing centre of gravity problem at extremities

\section{EXPERIMENT}

In this section, we present some experimental set up and evaluations done. Similarity of words are determined based on context. So, it is rather difficult to say which words are similar without a context. But if they belong to same parts of speech then we can assume that the contextual appearance of the words will also be similar if not exactly the same. Here, we show this with our experimental results.

\section{A. Generation of data set}

Unfortunately, there is no benchmark data set computing word similarity. Generally people have evaluated their word similarities [18], [19] using some kind of multiple choice question and their answers in TOEFL (Test of English as a Foreign Language) or $\mathrm{ESL}^{2}$ (English as a second language) or any other linguistic exams. Thus, for our work, we too prepared our data set from ESL mock exams. Like [4] we did not restrict ourselves only to verbs, rather we covered all four main parts of speech which we encountered in the multiple choice question in ESL. Each practice exams had 31 questions; with 4 choices. Out of these four, one gives you the exact match. Like Microsoft Research paraphrase corpus, [20] we assigned binary judgements to the word pair similarity instead of sentence similarity as mentioned in the data set. For the correct choices, according to ESL answer set, we assigned 1 as relevancy score of the word pair similarity, for the rest incorrect answers, we assigned 0 . We deliberately chose antonyms for the incorrect choices (out of 3 incorrect choices) except for the questions which

${ }^{2}$ https://www.esl.org/ 
TABLE II

SNAPSHOT OF DATA SET GENERATED FROM ESL MULTIPLE CHOICE QUESTIONS

\begin{tabular}{c|c|c|c}
\hline Relevance & word_1 & word_2 & POS \\
\hline 1 & balmy & warm & adjective \\
0 & balmy & cold & adjective \\
1 & pored & examined & verb \\
0 & pored & memorized & verb \\
1 & intersection & crossing & noun \\
0 & intersection & ending & noun \\
1 & repugnant & disgusting & adjective \\
0 & repugnant & delightful & adjective \\
1 & diligence & dedication & noun \\
0 & diligence & ease & noun \\
0 & widespread & limited & adjective \\
1 & harsh & extreme & adjective \\
0 & harsh & pleasant & adjective \\
1 & touched & began & verb \\
\hline
\end{tabular}

had some other options. In linguistic sense, we cannot really determine any crisp boundary for word similarity. The binary scores are assigned as a benchmark. We also found that for some words, which returned 'incorrect choice' in ESL test set, has some degree of similarity linguistically, might not be very relevant to the context of the question being asked. So, for these words, there are false negatives. We also assigned respective parts of speech for the word pairs. Here, in table.II we present a snapshot of the dataset we generated from ESL multiple choice questions.

\section{B. Evaluation strategy}

In this work, we present comparisons of evaluations of path measure, gloss overlap and our PGMeasure. For this we define the following metrices [21] to highlight the performance of the methods by changing the parameters from sentence level to word level. These methods are: Recall is a proportion of correctly predicted similar word pairs compared to all similar word pairs. Precision is a proportion of correctly predicted similar word pairs compared to all predicted similar word pairs. Rejection is a proportion of correctly predicted dissimilar word pairs compared to all dissimilar word pairs. Accuracy is a proportion of all correctly predicted word pairs compared to all word pairs. F-Measure (F1) is a uniform harmonic mean of precision and recall. Lastly, we define $\mathbf{f 1}$ as a uniform harmonic mean of rejection and recall. A scoring threshold for similar pairs is defined at 0.5 [21]. In this work, we include rejection and $f 1$ metrics in addition to the standard precision- recall based metrics as it presents another aspect of the performance based on the tradeoff between true positive and true negative judgments.

\section{Results}

1) Comparison of different senses of word pairs using different methods: We show here some of the word pair similarities at each sense level. Table.III illustrates some of the results we obtained from the data set generated. In some words, gloss overlap is 0 , which signifies that semantically they do not share any information, but taxonomically they are
TABLE III

WORD SIMILARITY RESULTS AT SENSE LEVEL

\begin{tabular}{c|c|c|c}
\hline (w_i\#s_k,w_j\#s_l) & $\mathrm{P}$ & $\mathrm{G}$ & $\mathrm{PG}$ \\
\hline (enquiry\#1,investigation\#1) & 0.06 & 0.25 & 0.30 \\
(enquiry\#1,investigation\#2) & 0.01 & 0.0 & 0.30 \\
(enquiry\#2,investigation\#1) & 0.48 & 0.0 & 0.30 \\
$\ldots$. & & & \\
(enquiry\#1,interview\#1) & 0.46 & 0.2 & 0.30 \\
(enquiry\#1,interview\#2) & 0.06 & 0.0 & 0.30 \\
$\ldots$ & & & \\
(outlets\#1,stores\#1) & 1.41 & 0.33 & 0.62 \\
(outlets\#1,stores\#2) & 0.06 & 0.0 & 0.30 \\
$\ldots$ & & & \\
\hline
\end{tabular}

TABLE IV

COMPARISON OF DIFFERENT EVALUATION PARAMETERS OF JC PATH MEASURE, GLOSS OVERLAP AND PG MEASURE USING TRIANGULAR MEMBERSHIP FUNCTION

\begin{tabular}{c|c|c|c|c|c|c}
\hline & Recall & Precision & F1 & Accuracy & Rejection & f1 \\
\hline P & 0.2 & 1 & 0.33 & 0.61 & 0.51 & 0.29 \\
G & 0.16 & 1 & 0.28 & 0.59 & 0.51 & 0.24 \\
PG & 0.2 & 1 & 0.08 & 0.53 & 0.51 & 0.29 \\
\hline
\end{tabular}

in some hierarchial level of some similarity. Our PGMeasure combines these kinds of information which we can see from this table. The tuning of membership functions can definitely provide us with more refined similarity values for the word pairs.

2) Comparison of performance of different similarity methods: In tables IV to VII, we present comparisons of different evaluation parameters like recall, precision, Fmeasure (F1), accuracy, rejection and $\mathrm{f} 1$ for the word pair similarities using path measure, gloss overlap and our proposed PGMeasure. In this case, to measure path measure of two given words, we have taken maximum similarity score of the synset pairs to represent the overall similarity of a word pair. Likewise for gloss overlap. We have shown the effects of variation in performance due to different membership functions used as well as different path based measures. The combination of Lin's similarity score as path measure with triangular membership function for the output fuzzy set has given us the better performance with higher accuracy, precision, recall and F1 measure. Then is Jiang's mode with triangular membership function in output. Due to extremity problem with trapezoidal membership, we can see the difference in performance in tables VI and VII.

TABLE V

COMPARISON OF DIFFERENT EVALUATION PARAMETERS OF LIN PATH MEASURE, GLOSS OVERLAP AND PG MEASURE USING TRIANGULAR MEMBERSHIP FUNCTION

\begin{tabular}{c|c|c|c|c|c|c}
\hline & Recall & Precision & F1 & Accuracy & Rejection & f1 \\
\hline P & 0.44 & 0.61 & 0.51 & 0.59 & 0.37 & 0.40 \\
G & 0.16 & 1 & 0.28 & 0.59 & 0.51 & 0.24 \\
PG & 0.32 & 0.73 & 0.44 & 0.61 & 0.45 & 0.37 \\
\hline
\end{tabular}


TABLE VI

COMPARISON OF DIFFERENT EVALUATION PARAMETERS OF JC PATH MEASURE, GLOSS OVERLAP AND PG MEASURE USING TRAPEZOIDAL MEMBERSHIP FUNCTION

\begin{tabular}{c|c|c|c|c|c|c}
\hline & Recall & Precision & F1 & Accuracy & Rejection & f1 \\
\hline P & 0.2 & 1 & 0.33 & 0.61 & 0.51 & 0.29 \\
G & 0.16 & 1 & 0.28 & 0.59 & 0.51 & 0.24 \\
PG & 0.04 & 1 & 0.08 & 0.53 & 0.51 & 0.07 \\
\hline
\end{tabular}

TABLE VII

COMPARISON OF DIFFERENT EVALUATION PARAMETERS OF LIN PATH MEASURE, GLOSS OVERLAP AND PG MEASURE USING TRAPEZOIDAL MEMBERSHIP FUNCTION

\begin{tabular}{c|c|c|c|c|c|c}
\hline & Recall & Precision & F1 & Accuracy & Rejection & f1 \\
\hline P & 0.44 & 0.61 & 0.51 & 0.59 & 0.371 & 0.40 \\
G & 0.16 & 1 & 0.28 & 0.59 & 0.51 & 0.24 \\
PG & 0.08 & 0.5 & 0.14 & 0.51 & 0.47 & 0.13 \\
\hline
\end{tabular}

\section{WordNet coverage and pitfalls}

The effectiveness of linguistic measures depends on a heuristic to compute semantic similarity between words as well as the comprehensiveness of the lexical resource. As WordNet is used as a primary lexical resource in this study, its comprehensiveness is determined by the proportion of words in the text collections that are covered by its knowledge base. In general, a major criticism of WordNet-based similarity measures is in its limited word coverage to handle a large text collection, particularly on the named entities coverage. The percentage of word coverage in WordNet decreases as the size of test collection and vocabulary space increases. Thus, the effectiveness of linguistic measures is likely to be effected because word-to-word similarity calculation will inevitably produce many "misses". One solution is to resort to approaches that utilize other knowledge resources, such as Wikipedia [22] or web search results [23], to derive semantic similarity between words.

\section{CONCLUSION}

We have presented a new measure of semantic relatedness based on combination of path based measures and gloss overlaps using fuzzy inference system. A pair of words is assigned a value of similarity based on the output of the FIS which is a combined result of the words' taxonomical location in the WordNet hierarchy as well as the overlap of words in their glosses. Our evaluation shows that this measure relates to human judgements and are reasonably correlated. Due to limitations in WordNet, there were many words which were not a valid entry in its database; as a result of which there is effect on the overall performance as well. Besides this, the difference in membership functions of the output fuzzy set has a great impact on the final similarity results of the word pairs. In this case, we found triangular membership function performs better than trapezoidal using Lin's method; so tuning and choosing proper methods for similarity computation are very essential criteria for this case. In future, we aim to use some machine learning algorithm to optimize the membership functions .

\section{REFERENCES}

[1] C. Fellbaum, WordNet: An Electronic Lexical Database. Bradford Books, 1998.

[2] G. Grefenstette, "Evaluation techniques for automatic semantic extraction: comparing syntactic and window based approaches," in Proc. of the SIGLEX Workshop on Acquisition of Lexical Knowledge from Text, Columbus Ohio, 1993.

[3] C. Gasperin, P. Gamallo, A. Agustini, G. Lopes, and V. Lima, "Using syntactic contexts for measuring word similarity," in Proceedings of the Workshop on Semantic Knowledge Acquisition and Categorisation. Citeseer, 2001.

[4] D. Yang and D. Powers, "Word similarity on the taxonomy of WordNet."

[5] M. Quillian, "Word concepts: A theory and simulation of some basic semantic capabilities," Behavioral Science, vol. 12, no. 5, pp. 410-430, 1967.

[6] B. BERANEK, N. I. C. MASS, and M. Quillian, "THE TEACHABLE LANGUAGE COMPREHENDER: A SIMULATION PROGRAM AND THEORY OF LANGUAGE," 1969.

[7] R. Rada, H. Mili, E. Bicknell, and M. Blettner, "Development and application of a metric on semantic nets," IEEE transactions on systems, man and cybernetics, vol. 19, no. 1, pp. 17-30, 1989.

[8] Z. Wu and M. Palmer, "Verbs semantics and lexical selection," in Proceedings of the 32nd annual meeting on Association for Computational Linguistics. Association for Computational Linguistics Morristown, NJ, USA, 1994, pp. 133-138.

[9] C. Leacock and M. Chodorow, "Combining local context and WordNet similarity for word sense identification," WordNet: An electronic lexical database, vol. 49, no. 2, pp. 265-283, 1998.

[10] P. Resnik, "Using information content to evaluate semantic similarity in a taxonomy," in International Joint Conference on Artificial Intelligence, vol. 14. Citeseer, 1995, pp. 448-453.

[11] J. Jiang and D. Conrath, "Semantic similarity based on corpus statistics and lexical taxonomy," Arxiv preprint cmp-lg/9709008, 1997.

[12] D. Lin, "Using syntactic dependency as local context to resolve word sense ambiguity," in ANNUAL MEETING-ASSOCIATION FOR COMPUTATIONAL LINGUISTICS, vol. 35. ASSOCIATION FOR COMPUTATIONAL LINGUISTICS, 1997, pp. 64-71.

[13] M. Lesk, "Automatic sense disambiguation using machine readable dictionaries: How to tell a pine cone from an ice cream cone," in Proceedings of the 5th annual international conference on Systems documentation. ACM New York, NY, USA, 1986, pp. 24-26.

[14] S. Banerjee, "Adapting the Lesk algorithm for word sense disambiguation to WordNet," Ph.D. dissertation, Citeseer, 2002.

[15] E. Mamdani and S. Assilian, "An experiment in linguistic synthesis with a fuzzy logic controller," International Journal of Man-Machine Studies, vol. 7, no. 1, pp. 1 - 13, 1975. [Online]. Available: http://www.sciencedirect.com/science/ article/B6WGS-4T73MHB-2/2/dbafdc3cee4f8c37f68b4d698b71728f

[16] M. Sugeno, Industrial applications of fuzzy control. Elsevier Science Inc. New York, NY, USA, 1985.

[17] A. Chong, T. D. Gedeon, K. W. Wong, and L. T. Kóczy, "A histogrambased rule extraction technique for fuzzy systems," in FUZZ-IEEE, 2001, pp. 638-641.

[18] T. Landauer and S. Dumais, "A solution to Plato's problem: The latent semantic analysis theory of acquisition, induction, and representation of knowledge," Psychological review, vol. 104, no. 2, pp. 211-240, 1997.

[19] D. Tatsuki, "Basic 2000 Words-Synonym Match 1," Interactive JavaScript Quizzes for ESL Students, http://www. aitech. ac. jp/ iteslj/quizzes/js/dt/mc-2000-01syn. html, 1998.

[20] B. Dolan, C. Quirk, and C. Brockett, "Unsupervised construction of large paraphrase corpora: Exploiting massively parallel news sources," in Proceedings of the 20th international conference on Computational Linguistics. Association for Computational Linguistics Morristown, NJ, USA, 2004

[21] P. Achananuparp, X. Hu, and X. Shen, "The evaluation of sentence similarity measures," in DaWaK08: Proceedings of the 10th international conference on Data Warehousing and Knowledge Discovery. Springer, pp. 305-316. 
[22] S. Ponzetto and M. Strube, "Knowledge derived from Wikipedia for computing semantic relatedness," Journal of Artificial Intelligence Research, vol. 30, no. 1, pp. 181-212, 2007.

[23] M. Sahami and T. Heilman, "A web-based kernel function for measuring the similarity of short text snippets," in Proceedings of the 15th international conference on World Wide Web. ACM, 2006, p. 386. 